\title{
Shape Optimization of the Active Element for Vibration Energy Harvesting
}

\author{
Rimvydas GAIDYS*, Darius ŽIŽYS*, Paulius SKĖRYS**, Audronė LUPEIKIENË*** \\ *Kaunas University of Technology, Studentu g. 56-344, Kaunas, Lithuania, E-mail: Rimvydas.Gaidys@ktu.lt \\ *Kaunas University of Technology, Studentu g. 56-344, Kaunas, Lithuania, E-mail: Darius.Zizys@Yahoo.com \\ **Siauliai University, Vilniaus g. 141, Šiauliai, Lithuania, E-mail: skeryspaulius@gmail.com \\ ***Vilnius University, Akademijos g. 4-625, Lithuania, E-mail: audrone.lupeikiene@mif.vu.lt \\ cross ${ }^{\text {ref }}$ http://dx.doi.org/10.5755/j01.mech.26.4.25253
}

\section{Introduction}

Recently the use of renewable energy has sky rocketed all around the globe. In the EU, it is $16 \%$ of all energy with plans for 2020 to get as high as $20 \%$ [1]. If we are only speaking about electricity found from renewable sources, then the statistics are even higher. In the EU in 2004, 14\% of electrical power was produced from sustainable sources of energy, and by 2016 that number was $28 \%$. [2]. Within just over 10 years' renewable energy production has increased twice. It should be noted that the sustainable energy generation techniques typically have many drawbacks, such as storage and transportation of that energy is only possible in electrical form which is difficult and inefficient to do, therefore the coal and oil can be shipped effectively and used where and when needed.

Of course, not only macro energy is increasing but also micro energy with its sources became the key to $21^{\text {st }}$ century. With consumer electronic devices getting even hungrier for electricity, certain saving techniques are developing. Semiconductor power levels have dropped significantly nowadays, mostly using milliwatts when functioning, and standby consumption is close to zero.

Appropriately of the serious shrinkage of power expenditure and the development of ultra-energy-saving devices, networks of sensors grow into reality and eliminated most previous disadvantages. Electricity supply is the major drawback that remains to this day. A combination of battery and a generator of electricity would definitely enhance the period for changing batteries significantly or eliminate a need for replacement $[3,4]$. The sustainable sources of electricity usable with such devices may be mechanical, solar, radiation or thermal [5]. There are now many rising applications of energy harvesting technology. One of them is a transducer which can generate electricity from the relative displacement within the system or the mechanical strain. [6].

This research is directed to establish mathematical and computational models for optimizing the form of the piezoelectric vibration energy harvesters (VEH), maximizing extracted energy, evaluating the results obtained and experimentally confirming results.

The COMSOL Multiphysics was used for simulation. MATLAB was used for the mathematical calculations.

Several experiments have been conducted to verify the results of the theoretical investigation. The methodology used for optimization of the geometrical shape of the piezoelectric VEH resulted in an increase in its efficiency by $16 \%$.

\subsection{Piezoelectric transduction mechanism}

If mechanically stressed, a piezoelectric ceramic is electrically polarized, creating an electric charge on its surface [7]. Such charges can be obtained and using electrodes connected to the surface of the piezoelectric material normal to deformation vector.

Some scientists intend to build a model for estimating power output that can be developed when a cantilever beam that has piezoelectric components attached is vibrating [8]. Some incorporate topology optimization methods to optimize VEH output voltage with quasi-static control requirements $[9,10]$, using techniques of topology optimization to increase the VEH's factor in electromechanical coupling working in higher vibration modes [11] or implemented an L-shaped generator of energy and tackled the limited bandwidth drawback $[12,13]$. Experimentally verified modelling results show that a VEH can operate efficiently from 10 to $18 \mathrm{~Hz}$ with a power density of 65.74 $\mu \mathrm{W} / \mathrm{cm}^{3}$ at $10 \mathrm{~Hz}$ and $341.68 \mu \mathrm{W} / \mathrm{cm}^{3}$ at $18 \mathrm{~Hz}$ [14].

The mechanism for piezoelectric transduction has quite high efficiency $(5-20 \%)$ [15]. Nonetheless, since the output is greatest when the piezoelectric VEH type cantilever operates in resonance mode, the fundamental transducer frequency must suit the ambient vibrations frequency [16, 17]. Due to the rarely stable frequency of ambient vibrations, this is one of the major shortcomings to tackle.

\subsection{Numerical model and methods analysis}

There was already a concept for a piezoelectric human motion impact-driven energy generator [18]. The layout of the system is a cantilevered beam among layers of PZT-5A with an added mass at the loose tip. Figure 1 displays a graphical description of the present model. $C$ stands for coefficient of damping, $K$ - stiffness, $M$ - the mass, $v(t)$ the voltage, $i(t)$ - current output and $F_{P}(t)$ - time varying applied force.

To research the VEH substratum thickness effect on performance with simulation the FE method was introduced. [19]. Electrical, frequency and mechanical analysis have been executed to assess structures' energy collection efficiency (with different thicknesses of a steel substratum). Piezoelectric single-crystal PMN material was used. Shown design has a piezoelectric material applied on all length of the cantilever. Therefore, similar concept generates greater output voltage with decreased piezoelectric zone size [18, 20]. 


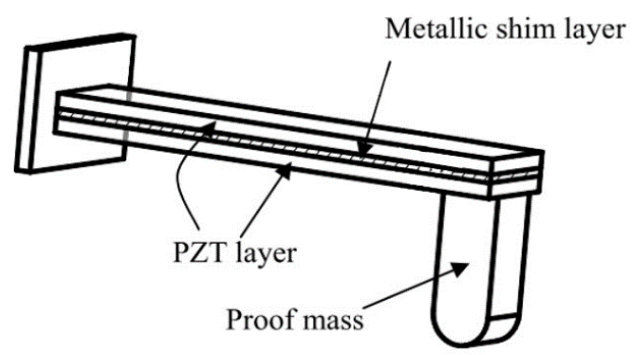

a

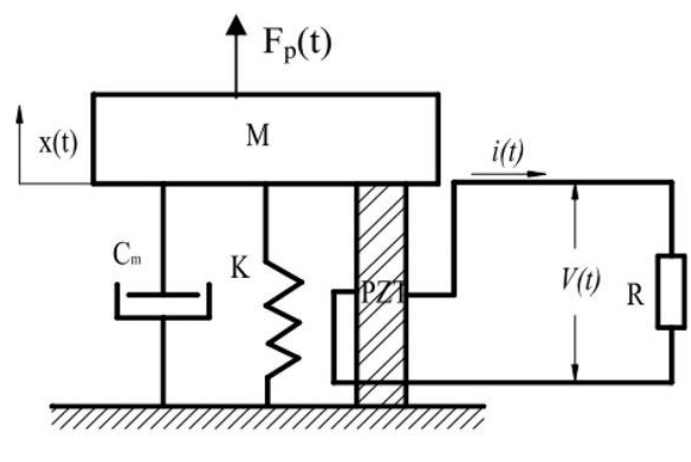

b

Fig. 1 Piezoelectric impact driven power generator: a) Piezoelectric bimorph schematic diagram; b) Schematic lumped system piezoelectric VEH electromechanical model [18]

Various numerical methods for solving the optimization problem were created. Table 1 summarizes some of the popular methods. Optimization techniques have different adaptations but interest in mathematical optimization is greatest if the process of experimentation is too costly or if the equations are too complex to solve without optimization [21].

It is clear that when the active element of the VEH functions in resonance phase therefore increases vibration amplitudes tremendously as well as power production. Many fundamental vibration modes have strain nodes in which the usual field of strain changes the sign towards the cantilever length. The paper [22] focuses on a dimensionless numerical transient study of a constant cross-section cantilever and an efficiently formed cantilever for the same first eigen frequency and minimum mass, with a view to determining the location of a strain node.

The impact-coupling configuration is examined in [23]. The active element of the VEH has a different lower and higher natural frequency ratio. Energy output of various frequency enhancement pairs with appropriate resistive values have a connection between lower and higher power output and natural frequencies as well as the position of the point of impact.

\section{Modelling shape optimization of the active element of VEH}

Frequency-up-conversion can be used to maximize the mechanical/electrical power output of the piezoelectric mechanical VEH. Another way to achieve that is mathematical optimization method. The VEH shape can be changed by maximization of the normal strain in the PZT layers with constraints to the geometrical (design) and physical (state) parameters. The problem of piezoelectric bimorph shape optimization was formulated and the dynamic problem was solved using equivalent static load technique.

\subsection{Electromechanical coupling}

The electromechanical conversions can be written as shown at Eqs. (1) and (2) for the linear piezoelectric material. The equations combine a piezoelectric constant stress with electrical displacement and permittivity or vector in conformity with and electrical field vector. In the Eqs. (1) and (2), the piezoelectrical element is used not as a sensor/generator but as an actuator.

$$
\begin{aligned}
& \varepsilon_{i}=S_{i j}{ }^{E} \sigma_{j}+d_{m i} E_{m}, \\
& D_{m}=d_{m i} \sigma_{i}+\zeta_{i k}{ }^{\sigma} E_{k},
\end{aligned}
$$

where: $i, j=1 \ldots 6$ and $m, k=1 \ldots 3$ correspond to various directions within the material coordinate system [24]. The purpose of this work is the VEH that operates in a generator mode, these equations should be rewritten:

$$
\begin{aligned}
& \varepsilon_{i}=S_{i j}{ }^{D} \sigma_{j}+g_{m i} D_{m}, \\
& E_{i}=g_{m j} \sigma_{i}+\beta_{i k}^{\sigma} D_{k},
\end{aligned}
$$

where: $\sigma$ is a stress $\left(\mathrm{N} / \mathrm{m}^{2}\right)$; $\varepsilon$ is a strain vectors; $\xi$ is permittivity $(\mathrm{F} / \mathrm{m}) ; E$ is a vector of applied electric field $(\mathrm{V} / \mathrm{m}) ; S$ is a matrix of compliance coefficients $\left(\mathrm{m}^{2} / \mathrm{N}\right) ; D$ is a vector of electric displacement $\left(\mathrm{C} / \mathrm{m}^{2}\right) ; d$ is a matrix of piezoelectric strain constants $(\mathrm{m} / \mathrm{V}) ; g$ is a matrix of piezoelectric constants $\left(\mathrm{m}^{2} / \mathrm{C}\right) ; \beta$ is a permittivity component $(\mathrm{m} / \mathrm{F})[13]$.

The $d_{31}$ mode is shown in Fig. 2. Index $i$ in $d_{i j}$ marks the axis along which the voltage is generated ( $\mathrm{z}$-axis is number 3). Index $j$ marks the direction of stress application (xaxis is number 1 ).

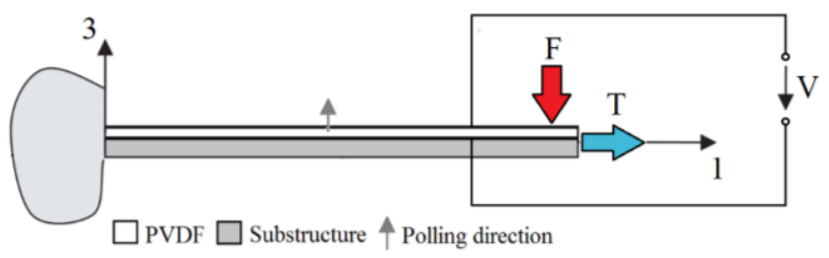

Fig. 2 Scheme of the PZT material operated in $d_{31}$ mode

The constitutive Eqs. (3) and (4) can be simplified to for $d_{31}$ mode:

$$
\left\{\begin{array}{l}
S_{1}=S_{11}^{E} \cdot \sigma_{1}+d_{31} \cdot E_{3} \\
D_{3}=d_{31} \cdot \sigma_{1}+\varepsilon_{33}^{T} \cdot E_{3}
\end{array}\right. \text {. }
$$

The electromechanical conversion of the piezoelectric VEH operating in $d_{31}$ mode is defined in all the mentioned Eqs.

To express piezoelectric equations for the FE method, differential equation can be written in matrix form. 
List of numerical optimization methods with constrains [25]

\begin{tabular}{|c|c|c|c|}
\hline & Classification & Algorithm & Remarks \\
\hline \multirow{8}{*}{$\begin{array}{l}\text { Direct } \\
\text { method }\end{array}$} & \multirow{4}{*}{ Primal method } & Sequential linear programming (SLP) & \multirow{4}{*}{$\begin{array}{l}\text { A solution of an approximated subproblem is ob- } \\
\text { tained and the optimum solution is found in an } \\
\text { iterative process. Generally used in engineering } \\
\text { optimization. }\end{array}$} \\
\hline & & Gradient projection method (GRP) & \\
\hline & & Feasible directions method (FDM) & \\
\hline & & $\begin{array}{l}\text { Sequential or recursive quadratic method (SQP or } \\
\text { RQP) }\end{array}$ & \\
\hline & \multirow{4}{*}{$\begin{array}{l}\text { Transformation } \\
\text { method }\end{array}$} & $\begin{array}{l}\text { Sequential unconstrained minimization technique } \\
\text { (SUMT) }\end{array}$ & \multirow{4}{*}{$\begin{array}{l}\text { A constrained problem is transformed to an un- } \\
\text { constrained problem and the solution is found in } \\
\text { an iterative manner. }\end{array}$} \\
\hline & & Penalty function method & \\
\hline & & Barrier function method & \\
\hline & & Augmented Lagrangian method & \\
\hline $\begin{array}{l}\text { Indirect } \\
\text { method }\end{array}$ & Hybrid method & Optimality criteria method & $\begin{array}{l}\text { The KKT conditions of the formulated problem } \\
\text { are used to drive an iterative solution strategy. It } \\
\text { has been verified to be numerically inefficient. } \\
\text { Rarely used except for topology optimization } \\
\text { methods. }\end{array}$ \\
\hline
\end{tabular}

$$
\begin{aligned}
& M \ddot{z}+C_{z z} \dot{z}+K_{z z} z+K_{z \phi} \phi=F D, \\
& K_{z \phi}^{t} z+K_{\phi \phi} \phi=Q,
\end{aligned}
$$

here: $z$ is a nodal point displacement vectors, $\dot{z}$ is a nodal point velocity and $\ddot{z}$ is an acceleration vector of nodal point respectively. The subscript $\phi$ is the electrostatic potential and refers to electrical quantities, it is a scalar. The subscript $z$ refers to mechanical quantities. So, the combination of these two refers to electromechanical coupling matrices. $C$, $K$ and $M$ are global matrices and $\Phi, F, Z$ and $Q$ are denote vectors.

\subsection{General shape optimization problem}

Optimization is a research area that mostly concentrates on the numerical method of the following mathematical design issue [25].

$$
\begin{aligned}
& \text { Find } b \in R^{n}, \\
& \text { to optimize } f(b), \\
& \text { subject to } h_{i}(b)=0, i=1, \ldots, l, \\
& g_{i}(b) \leq 0, j=1, \ldots, m, \\
& b_{L} \leq b \leq b_{U},
\end{aligned}
$$

here: $b$ is the design variable vector with $n$ elements, $f$ is the objective function, $h_{i}$ is the $i^{\text {th }}$ equality constraint, $g_{j}$ is the $j^{\text {th }}$ inequality constraint, $b_{L}$ and $b_{u}$ are limit conditions for $b$, respectively, $l$ is the number of equality constraints and $m$ is the number of inequality constraints. Depending on the problem to minimize or to maximize a specific design parameter is the main goal of optimization procedure [26]. The objective function (the parameter targeted for improvement) is written as shown in Eq. (9), with limit conditions as written in Eqs. (10) to (12).

\subsubsection{Structural optimization}

The state equation is computationally resolved and the solution is used for objective purpose and limitations assessment [25]. In Eq. (10), equality constraints are regarded as the governing Eq. (10). formulated.

Structural optimization under static loads can be

$$
\begin{aligned}
& \text { Find } b \in R^{n}, \\
& \text { to optimize } f(b, z), \\
& \text { subject to } K(b) z=F, \\
& g_{j}(b, z) \leq 0, j=1, \ldots, m, \\
& b_{L} \leq b \leq b_{U},
\end{aligned}
$$

The volume of the structure or a specific response can be the objective function in (14). The equality constraint (15) generally is equation of state. The limit values on strains, displacements and other state variables generally are defined by the inequality constraints in (16).

\subsubsection{Structural optimization under dynamic loads}

The equation of state for FEM can be described as Eq. (15) in the case of a linear static problem. In Eq. (15), $b$ is the design variable vector (usually describing geometry); $F$ is external load vector; $K$ is the stiffness matrix and $z$ is the vectors of nodal displacements [25]. Dynamic loads show a time depended state of the structure and its response to the external load. As a consequence, a time - specific response in optimization equations should be implemented to represent the dynamics. Although all loads are dynamic, most can be reduced to static load. And those that are not simplified to static are solved by optimizing the dynamic response. Dynamic response optimization depends on time and computation consummation, so static charges are usually favoured [10]. If $F(t)$ is an external dynamic load vector, the equation of state in the FEM formulation is:

$$
M(b) \ddot{z}+K(b) z=F(t)
$$

where: $\ddot{z}$ is the acceleration nodal point vector and $M$ is the mass matrix. Then the optimization problem for dynamic response can be described by Eqs. (19) to (23). 
Find $b \in R^{n}$,

to optimize $f(b, z, t)$,

subject to $M(b) \ddot{z}+K(b) z=f(t)$;

$g_{j}(b, z, t) \leq 0, j=1, \ldots, m$,

$b_{L} \leq b \leq b_{U}$

The method of achieving the design solution of Eqs. (19) to (23) is dynamic response optimization. The independent time variable exists in the functions and therefore problem is different from static response optimization. Eq. of state (21) can be solved using one of the methods of the numerical integration of the ordinary differential equations and restrictions in Eqs. (21) and (23) for each of the discrete steps should be established. This shows the above disadvantage, that a large amount of time-based constraints has to be dealt with. Through various methods, such as peak value estimation or time domain integration, this issue can be addressed, but [25] these methods do not significantly reduce complexity levels. Dynamic response optimization with large-scale problems is therefore not suitable. Furthermore, it can be said that translating the dynamic equation to a static equation is more convenient then optimizing it since there is an iterative charge issue.

Fig. 3 illustrates the scheme of the active element of the mechanical VEH for the optimization the elastic normal strain in the layers. It is possible to express and formulate the mathematical problem solution shown below in Eqs. (24) to (27).

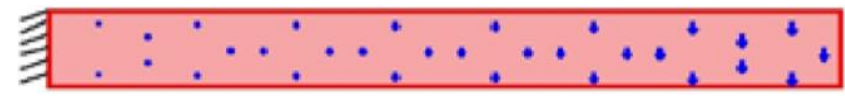

Fig. 3 Scheme of the active element of the mechanical VEH. Boundary condition and external load. Blue marks volume forces

The objective function can be written as Eq. (24) to solve the reduced dynamic problem (the transient analysis of a cantilevered type $\mathrm{VEH}$ ) in statics:

$$
\max Z=\int_{0}^{L}\left|\frac{d u}{d x}\right| d x .
$$

In this case the Eq. of state is expressed as:

$$
[K]\{U\}=\{F\} \text {. }
$$

Constrains to the thickness of the active element Eq. (26) and the design variables are as written in Eq. (27):

$$
\begin{aligned}
& H_{\min } \leq q_{0}+q_{1} x^{1}+q 2 x^{2}+q_{3} x^{3} \leq H_{\max }, \\
& {[Q]=\left(q_{0}, q_{1}, q_{2}, q_{3},\right),}
\end{aligned}
$$

where: Eq. (25) is equation of state, $H_{\min }$ and $H$ are the lower and upper constraint to thickness of the active element, $Q$ is the design variable, $K$ is a stiffness matrix and $U$ is a vector of displacements, $L$ is length of the active element, $F$ is external forces factor, objective function $Z$ is an integral value of the elastic strains on the active layer along length).

\subsection{Results and analysis of the optimal shape of the VEH active element}

The intention of the piezoelectric VEH active element optimization was to maximize the elastic normal strain field on the layer of the active element when it is subjected to equation of state (25) with constraints to the geometric parameters of the active element (the element's thickness).

The voltage and power output should be increased with piezoelectric material to this surface attached. The geometric parameters were restricted so that the active element during the optimization process did not become thinned or thickened unreasonably [27]. The dynamic calculation is transformed into a static one and optimization is done iteratively. A dynamic load is split into numerous static loads during optimization procedure and a composition with multiple loading conditions is optimized. The Nelder-Mead numerical method used to find the maximum of an objective function.

The original shape of the bimorph active element is given in Fig. 4. Comsol Multiphysics structural mechanics and optimization analysis modules were used in the solution. The rectangular first order Lagrange finite element approximation was used. An optimized geometry of the active element is shown in Fig. 4., the introductory boundaries of the active element are shown as a black contour.
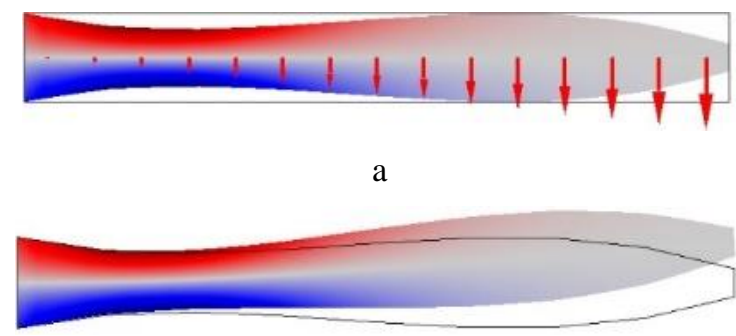

b

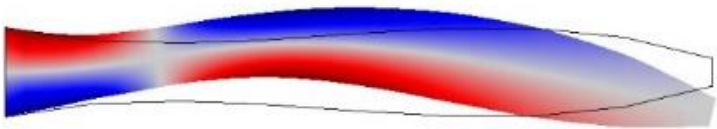

Fig. 4 An optimal shape active element, normal strain field: $a$ - initial and optimal shape of the active element, b - first transversal vibration mode of optimally shaped active element at $\omega_{1}=50 \mathrm{~Hz}, \mathrm{c}-$ second transversal vibration mode of optimally shaped active element at $\omega_{2}=369 \mathrm{~Hz}$

$\omega_{1}=50 \mathrm{~Hz}$ was the first eigen frequency for the optimally shaped element, the corresponding first eigen frequency of the constant cross-section active element was $\omega_{1}=66 \mathrm{~Hz}$.

$\omega_{2}=369 \mathrm{~Hz}$ was the second transversal vibration eigen frequency for the optimally shaped active element, the corresponding second eigen frequency of the constant crosssection active element was $\omega_{2}=389 \mathrm{~Hz}$.

The transient analysis was done after the modal study for the dynamic analysis of the optimal shape active 
element, with a time-depended force $F(t)$ acting on it. The force $F(t)$ was described as the active element volume force acting in the transverse direction and formalized as thickness/ force relation. In Fig. 5, a can be seen the integral value of normal strain in the top layer of the rectangular constant cross-section active element (or initial shape) and of the optimally shaped active element (Fig. 5, b) during a given time interval.

The peak of the normal strain created by an active element in optimal form is substantially higher and it is $3,15 \cdot 10^{-4} \mathrm{~m}$ while for constant cross section active element it is $2,11 \cdot 10^{-4} \mathrm{~m}$. During this analysis the load frequency corresponds to the first eigen frequency of constant and optimal shape active element and the same acceleration parameters impacted each active element. Because there is some difference in eigen frequency between constant and optimised active elements the criteria of effectiveness in Eq. (28) are derived.

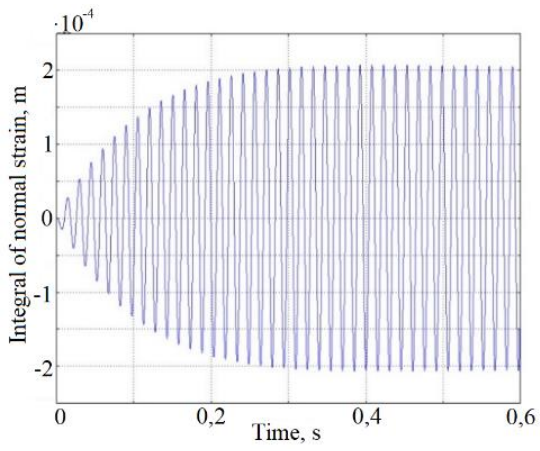

a

$$
E_{\text {eff }}=\omega_{1} \cdot \max \frac{d u}{d x}
$$

The constant cross-section active element effectiveness criteria is $E_{\text {eff }}^{\text {const }}=135,3 \cdot 10^{-4} \mathrm{~m} / \mathrm{s}$, the optimal shape active element effectiveness criteria is $E_{\text {eff }}^{\text {opt }}=157,5 \cdot 10^{-4} \mathrm{~m} / \mathrm{s}$, and the relative effectiveness is the ratio of these values $\frac{E_{e f f}^{\text {opt }}}{E_{\text {eff }}^{\text {const }}}=1,16$.

The shape of a VEH was optimized to maximize the integral of elastic normal strain on the top layer of the active element. After the dynamic analysis of the optimal and constant cross section active elements it is clear that integral value of normal strain in the top layer of the optimal shape active element of the VEH biggest output was increased by $49 \%$ and after adaptation of effectiveness criterion output was increased by $16 \%$.

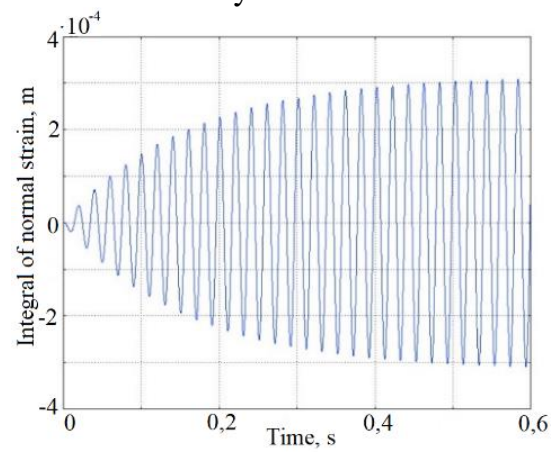

$\mathrm{b}$

Fig. 5 Dynamics analysis of the active element. The integral value of normal strain in the upper layer of the active element: (a) Constant cross-section active element; (b) Optimal shape active element

\section{Experimental verification}

The aim of the experimental studies was to verify the adequacy of the developed FE model and check the dynamic characteristics of the VEH active element.

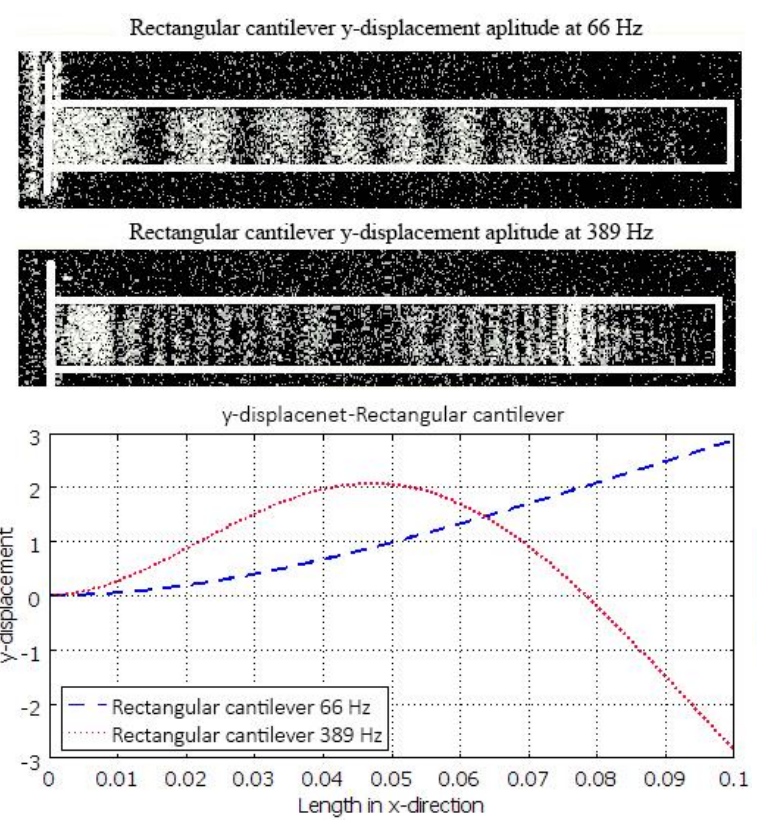

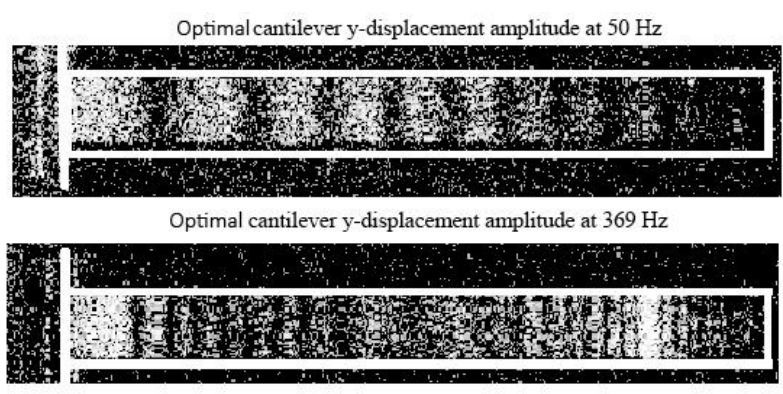

y-displacement-Optimal cantilever

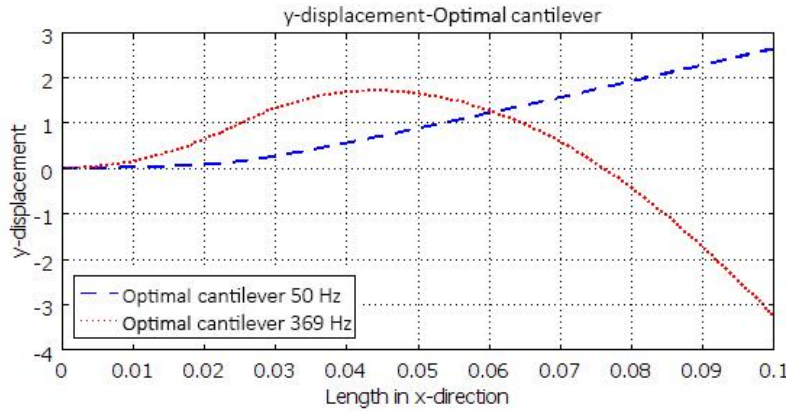

$\mathrm{b}$

Fig. 6 Patterns of interferometry fringes of cantilever type active element and corresponding eigen modes from numerical modal analysis: (a) Constant cross section active element; (b) Optimal shape active element 


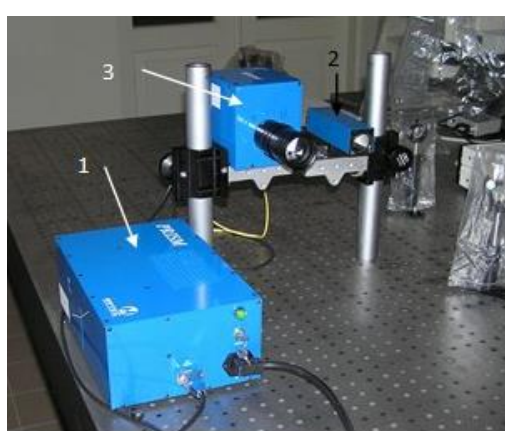

$\mathrm{a}$

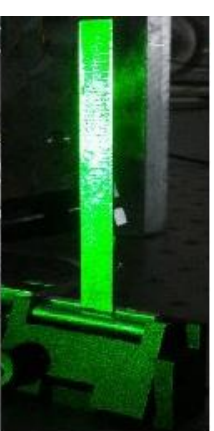

b

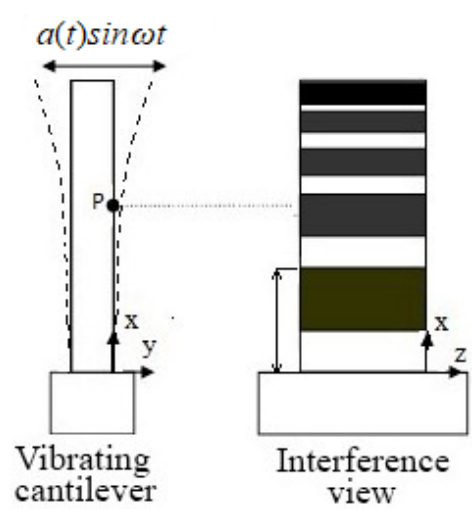

c

Fig. 7 PRISM Holography experimental setup: (a) The PRISM holography stand ( 1 - control block; 2 - object beam source; 3 - camera); (b) The rectangular active element specimen; (c) Interferometric view of vibrating active element

For the development of rectangular and optimally shaped cantilevers were manufactured for the experiment. It was done by water jet cutting the active elements.

Different setups of active element were investigated in PRISM Holography experiment.

To verify the FE model, transverse vibration eigen frequencies of the active element had to be experimentally measured when active element was excited by kinematic harmonic load and compared with an eigen frequencies obtained from numerical modal analysis simulation. The holographic interferometry was done and patterns of interferometry fringes of cantilever type element's element are presented in Fig. 6. The experimental setup can be seen in Fig. 7. The experimentally and numerically obtained eigen forms of transversal vibration were compared by analysing bright and dark bands of this patterns. The zones with darker band correspond to higher displacement. Because the displacement in modal analysis is dimensionless this is sufficient for comparing experimental and simulation results.

The dark region (Fig. 6) represents the maximums of displacement, while the bright white lines represent the minimum displacement or nodal points with vibration amplitudes of zero. These nodal points correspond to the vibration amplitude minimums of the eigen mode in the modal analysis simulation.

\section{Conclusions}

1. The mathematical and numerical models of shape optimization problem were built to maximize the normal strain in the active layer of the VEH during transversal vibrations. By optimizing the shape of the active element, the maximum performance of the normal strain was risen by $49 \%$ in the active layer of the VEH. The rise in normal output was $16 \%$ higher compare to the power output from a constant cross-section active element following the implementation of the effectiveness criterion standardizing the value of the integral of normal strain with regard to excitation frequency.

2. The stands and methodologies for the experimental validation of the defined mathematical and numerical model were created. Data gathered from the experiment using the holographic interferometry method were contrasted with simulation eigen frequencies and the error does not exceed 3\%.

\section{References}

1. Mohsen, Raeis-Zadeh, S. 2011. Measurement of ion mobility in dielectric liquids, Diploma work in the Master Program Electric Power Engineering, Diploma work No. 66/2011.

2. World Energy Resources 2013 Survey, World Energy Council.

3. Beepy, S.; White, N. 2012. Energy harvesting for autonomous systems, IEEE Industrial Electronics Magazine 6,1 .

4. Beepy, S. P.; Tudor, M. J.; White, N. 2006. Energy harvesting vibration sources for microsystems applications, Measurement Science and Technology 17(12): 175-195.

https://doi.org/10.1088/0957-0233/17/12/R01.

5. Erturk, A.; Inman, D. J. 2009. Electromechanical Modelling of Cantilevered Piezoelectric Energy Harvesters, In Energy Harvesting Technologies: 41-77.

6. Donovan, J. 2016. New applications for Energy Harvesting.

7. Moheimani, S. O. R.; Fleming, A. J. 2006. Piezoelectric Transducers for Vibration Control and Damping, Advances in Industrial Control: 9-35.

8. Sodano, H. A.; Park, G.; Inman, D. J. 2004. Estimation of Electric Charge Output for Piezoelectric Energy Harvesting, Strain: An International Journal for Experimental Mechanics 40(2): 49-58.

9. Mello, L. A. M.; Kiyono, C. Y.; Nakasone, P. H., Silva, E. C. N. 2014. Design of quasi-static piezoelectric plate-based transducer by using topology optimization, Smart Materials and Structures 23(2). https://doi.org/10.1088/0964-1726/23/2/025035.

10. Zhang, X.; Kang, Z. 2014. Dynamic topology optimization of piezoelectric structures with active control for reducing transient response, Computer Methods in Applied Mechanics and Engineering 281: 200-219. https://doi.org/10.1016/j.cma.2014.08.011.

11. Nakasone, P. H.; Silva, E. C. N. 2010. Dynamic design of piezoelectric laminated sensors and actuators using topology optimization, Journal of Intelligent Material Systems and Structures 21(16): 1627-1652.

12. Mahmoudi, S. 2014. Enhancement of the performance of a hybrid nonlinear vibration energy harvester based on piezoelectric and electromagnetic transductions, Smart Material Structures 23. 
https://doi.org/10.1088/0964-1726/23/7/075024.

13. Erturk, A.; Renno, J.; Inman, A. 2009. Modeling of Piezoelectric Energy Harvesting from an L-shaped Beam-mass Structure with an Application to UAVs, Journal of Intelligent Material Systems and Structures 20, 5: 633-642. https://doi.org/10.1177/1045389X08098096.

14. Li, W.G.; He, S.; Yu, S. 2010. Improving power density of a cantilever piezoelectric power harvester through a curved L-Shaped proof mass, IEEE Transactions on Industrial Electronics, 57(3): 868-876.

15. Knight, C.; Behrens, S.; Davidson, J. 2008. Review energy options for wireless sensor nodes, Sensors 8, 12: 8037-8066. https://doi.org/10.3390/s8128037.

16. Leadenham, S.; Erturk, A. 2015. Unified nonlinear electroelastic dynamics of a bimorph piezoelectric cantilever for energy harvesting, sensing, and actuation, Nonlinear Dynamics 79(3): 1727-1743. https://doi.org/10.1007/s11071-014-1770-x.

17. Ashraf, K.; Khir, M. H. M.; Dennis, J. O.; Baharudin, Z. 2013. A wideband, frequency up-converting bounded vibration energy harvester for a low-frequency environment, Journal Smart Material and Structures 22(2). https://doi.org/10.1088/0964-1726/22/2/025018.

18. Sheng, W.; Hong, H.; Siyuan, H. 2013. Modelling and experimental investigation of an impact-driven piezoelectric energy harvester from human motion, Smart Materials and Structures 22(10). https://doi.org/10.1088/0964-1726/22/10/105020.

19. Sunithamani, S.; Lakshmi, P.; Eba, F. E. 2013. PZT length optimization of MEMS piezoelectric energy harvester with a non-traditional cross section: simulation study, Microsystem Technologies 20(12): 2165-2171. https://doi.org/10.1007/s00542-013-1920-y.

20. Djuguma, R.; Trivailo, P.; Graves K. 2009. A study of energy harvesting from piezoelectrics using impact forces, The European Physical Journal Applied Physics 48(1).

21. Pister, K. S. 1972. Mathematical modelling for structural analysis and design, Elsevier 18(3): 353-375. https://doi.org/10.1016/0266-8920(88)90025-2.

22. Žižys, D.; Gaidys, R.; Daukševičius, R.; Ostaševičius, V.; Daniulaitis, V. 2016. Segmentation of a vibro-shock cantilever-type piezoelectric energy harvester operating in higher transverse vibration modes, Sensors 16(1): 114. https://doi.org/10.3390/s16010011.

23. Žižys, D.; Gaidys, R.; Ostaševičius, V.; Narijauskaitè, B. 2017. Vibro-shock dynamics analysis of a tandem low frequency resonator-high frequency piezoelectric energy harvester, Sensors 1(5): 1-21. https://doi.org/10.3390/s17050970.

24. Kumar, A.; Sharma, A. R.; Kumar, R.; Vaish, V.; Chauhan, S. 2014. Finite element analysis of vibration energy harvesting using lead-free piezoelectric materials: A comparative study, Journal of Asian Ceramic Societies 2(2):138-143. https://doi.org/10.1016/j.jascer.2014.02.001.

25. Park, J. 2007. Analytic Methods for Design Practice, Springer Science \& Business Media.

26. Suzuki, Y.; Miki, D.; Edamoto, M.; Honzumi M. 2010. A mems electret generator with electrostatic levitation for vibration-driven energy-harvesting applications, Journal of Micromechanics and Microengineering 20. https://doi.org/10.1088/0960-1317/20/10/104002.

27. Dauksevicius, R.; Briand, D.; Quintero, A. V. 2013. Multiphysics finite element model of a frequency-amplifying piezoelectric energy harvester with impact coupling for low-frequency vibrations, Journal of Physics Conference Series 476. https://doi.org/10.1088/1742-6596/476/1/012090.

R. Gaidys, D. Žižys, P. Skèrys, A. Lupeikienė

\section{SHAPE OPTIMIZATION of THE ACTIVE ELEMENT FOR VIBRATION ENERGY HARVESTING}

S u m m a r y

As the use of renewable energy is increasing exponentially all around the world, the micro energy sources are no different. One of renewable micro energy generator is transducer which can make electricity from the relative displacement present within the system or the mechanical strain. A technique was created to maximize the collection of the electricity generated from a piezoelectric fibre, based on modes of transversal vibration. Created the mathematical model and of the dynamic element's shape advancement issue. Normal strain generation was expanded by $49 \%$. The relative energy rise was $16 \%$. The exploratory stands and strategies were created for the test confirmation of the depicted numerical modelling results. Comes about gotten from the try utilizing the holography method were compared to numerically gotten eigen frequencies and mode shapes, and the relative error does not surpass $3 \%$ of the eigen frequencies.

Keywords: piezoelectric transducers, modelling and simulation, piezoelectric transduction mechanism, energy harvester, shape optimization.

Received February 07, 2020

Accepted August 24, 2020 